\section{Phenotypic screening on target}

\section{By Lev Osherovich, Senior Writer}

U.S. researchers have used forward genetics, a combination of phenotypic screening and genetics, to identify a therapeutic for leukemia and its target, the metabolic enzyme nicotinamide phosphoribosyl transferase. ${ }^{1}$ The platform plugs a gap in phenotypic screens that typically
"One of the problems with phenotypic screens is that the target or mechanism of action is often unknown. Applying forward genetics is a powerful approach to addressing this roadblock in phenotypic screen development."

-Michael Wei, Stanford University School of Medicine leave users guessing how their molecules work.

The strategy contrasts with the conventional approach to finding drug targets by biochemical methods or by selecting for mutations that affect drug response.

"One of the problems with phenotypic screens is that the target or mechanism of action is often unknown," said Michael Wei, an instructor in the Department of Pediatrics at the Stanford University School of Medicine and a member of the team that reported on the new screening platform. "Applying forward genetics is a powerful approach to addressing this roadblock in phenotypic screen development."

\section{Unbiased search}

The team started with a target-agnostic phenotypic screen of 115,000 molecules from a commercial library to identify compounds that prevented growth of cultured acute lymphoblastic leukemia (ALL) cells at nanomolar concentrations. The most potent compound, STF-118804, decreased growth in a variety of ALL cell lines compared with vehicle.

In a mouse xenograft model of ALL, STF-118804 was well tolerated and improved survival.

To figure out how STF-118804 worked, the team transfected ALL cells with a small hairpin RNA library covering the entire genome and then exposed the transfected cells to STF-118804.

The team reasoned that cells expressing shRNAs against the actual target of STF-118804 would have a clear increase in death-from the combined effects of the small molecule and the shRNA-compared with cells expressing off-target shRNAs.

Indeed, high throughput sequencing of shRNA librarytransfected cells showed those that underwent knockdown of nicotinamide phosphoribosyl transferase (NamPRT; NAMPT) were underrepresented in drug-treated vs. vehicle-treated populations.

Wei said that the shRNA library's high level of genomic coverage, with over 25 shRNA clones per gene, made it an ideal screening tool for target discovery.

"This library has very high coverage of shRNAs per gene, giving us confidence in the recovery of genes. This allowed us to home in right away on the target of the drug."

The team went on to show that STF-118804 blocked NAMPT's catalytic activity in vitro. Results were reported in Chemistry \& Biology. A pending patent on STF-118804 is available for licensing from Stanford University.

The shRNA library and the methods were developed by coauthors Jonathan Weissman, a professor of cellular and molecular pharmacology at the University of California, San Francisco and an investigator in the Howard Hughes Medical Institute, and Michael Bassik, acting assistant professor of genetics at Stanford's School of Medicine. UCSF has filed patents on the use of the shRNA platform for identifying drug targets.

The study was led by Michael Cleary, a professor of pediatric cancer biology and of pathology at Stanford's School of Medicine.

\section{Amped about NAMPT}

Despite the elegant proof of concept for matching a compound to its target, it is unclear whether STF-1189804 is a good drug candidate or even whether NAMPT is a viable target.

NAMPT normally performs an enzymatic step in the synthesis of nicotine adenine dinucleotide $\left(\mathrm{NAD}^{+}\right)$, an electron-ferrying small molecule that is critical for cellular metabolism.

Two previous NAMPT inhibitors-Topotarget A/S's APO866 and a molecule tested by the EORTC ${ }^{2}$ - failed in clinical trials in various solid tumors.

In October, researchers at the Genentech Inc. unit of Roche reported preclinical biomarker studies with the company's own NAMPT inhibitors. ${ }^{3}$ Genentech spokeswoman Nadine Pinell said that the company and partner Forma Therapeutics Holdings LLC are now determining the next steps for the program.

The Stanford team showed that STF- 118804 caused in vitro NAMPT inhibition comparable to that for Topotarget's APO866 but did not report cell culture or in vivo data comparing the two compounds.

Thus, it is unclear how STF-118804 measures up against previous NAMPT inhibitors. The new compound does have a distinct structure and was tested in the hematological cancer setting rather than in solid tumors.

"We have some hypotheses about why leukemia cells should be especially sensitive to NAMPT" vs. healthy bone marrow, said Wei. "We didn't see significant toxicity in mouse models and saw a good therapeutic index when we look at diseased vs. normal bone marrow progenitors."

Wei thinks that bioavailability and toxicity issues will be easier to manage in hematological malignancies than in solid tumors.

"The Topotarget compound was tested in relapsed solid tumors, 
so we don't know if the indication is necessarily the best for NAMPT inhibition," said Wei. "They had to administer the drug as a 96-hour infusion, so we might be able to improve on that."

Wei said that his next step is to seek an industry partner to conduct detailed preclinical toxicity and efficacy studies with STF-118804.

Osherovich, L. SciBX 6(43); doi:10.1038/scibx.2013.1213

Published online Nov. 7, 2013

\section{REFERENCES}

1. Matheny, C.J. et al. Chem. Biol.; published online Oct. 31, 2013; doi:10.1016/j.chembiol.2013.09.014

Contact: Michael L. Cleary, Stanford University School of Medicine, Stanford, Calif.

e-mail: mcleary@stanford.edu
2. Hovstadius, P. et al. Clin. Cancer Res. 8, 2843-2850 (2002)

3. Shames, D.S. et al. Clin. Cancer Res.; published online Oct. 4, 2013; doi:10.1158/1078-0432.CCR-13-1186

COMPANIES AND INSTITUTIONS MENTIONED

EORTC, Brussels, Belgium

Forma Therapeutics Holdings LLC, Watertown, Mass.

Genentech Inc., South San Francisco, Calif.

Howard Hughes Medical Institute, Chevy Chase, Md.

Roche (SIX:ROG; OTCQX:RHHBY), Basel, Switzerland

Stanford University, Stanford, Calif.

Stanford University School of Medicine,

Stanford, Calif.

Topotarget A/S (CSE:TOPO), Copenhagen, Denmark

University of California, San Francisco, Calif. 